\title{
Post-Immunotherapy Robotic-Assisted Resection for Primary Anorectal Melanoma: A Case Report
}

\author{
Keita Tsutsui ${ }^{a}$ b Dai Ogata ${ }^{a}$ Shunsuke Tsukamoto ${ }^{c}$ Konosuke Moritanic \\ Taisuke Mori $^{d}$ Kenjiro Namikawa ${ }^{a} \quad$ Akira Takahashi $^{a}$ \\ Yukihide Kanemitsu ${ }^{c}$ Naoya Yamazaki ${ }^{a}$ \\ aDepartment of Dermatological Oncology, National Cancer Center Hospital, Tokyo, \\ Japan; ${ }^{b}$ Department of Dermatology, Fukuoka University, Fukuoka, Japan; 'Derpartment \\ of Colorectal Surgery, National Cancer Center Hospital, Tokyo, Japan; ${ }^{\mathrm{d}}$ Department of \\ Diagnostic Pathology, National Cancer Center Hospital, Tokyo, Japan
}

\section{Keywords}

Anorectal malignant melanoma - Abdominoperineal resection - Lymph node dissection · Immunotherapy · Pembrolizumab

\begin{abstract}
Anorectal malignant melanoma (AMM) is a rare and aggressive neoplasm. Here, we report a case of AMM that was treated with robotic-assisted abdominoperineal resection (APR) after immunotherapy. A 68-year-old Japanese woman presented at our hospital because of diagnosis AMM. Computed tomography revealed a mass in the lower anorectal region and enlarged perirectal lymph nodes, but showed no evidence of distant metastases. We determined that radical resection would be difficult; therefore, the patient received immunotherapy with pembrolizumab for nine cycles. The size of pararectal lymph nodes was reduced, and the patient subsequently underwent robot-assisted APR and lymph node dissection. Histopathological examination showed spindle-shaped atypical melanocytes with acidophilic changes indicative of tumor cell necrosis in the rectal mucosa. We found no viable tumor cells in all 48 lymph nodes that were resected, and resection margins were tumor free. The patient was able to complete 15 cycles of adjuvant immunotherapy with pembrolizumab and remained relapse free at the 2-year postoperative follow-up. The present case showed that combination of complete surgical resection and immunotherapy is expected to improve outcomes in AMM patients if immunotherapy is effective.
\end{abstract}

\section{Karger"}




\section{Introduction}

Anorectal malignant melanoma (AMM) is a rare disease that accounts for less than $1 \%$ of primary anorectal malignancies [1]. The prognosis for AMM is considered to be poor because it is often misdiagnosed as hemorrhoids and difficult to diagnose correctly, especially in the early stages. In particular, lymph node metastasis (LNM) associated with AMM correlates poor survival outcome. Thus far, a standardized therapeutic strategy to treat AMM with LNM has not been established [2]. Previous reports have described the effectiveness of immunotherapy for unresectable mucosal melanoma, including AMM9 [3], but few reports have been published on multimodal therapy for AMM combining immunotherapy and surgery. Here, we report a case of AMM with LNM that was treated with robotic-assisted abdominoperineal resection (APR) after immunotherapy.

\section{Case Report}

A 68-year-old Japanese woman presented at her local hospital with a complaint of melena. Colonoscopy revealed an elevated tumorous lesion within the lower rectum (Fig. 1) that was confirmed to be malignant melanoma based on the results of histopathological examination. No BRAF mutations were identified on genetic analysis. Computed tomography revealed a mass in the lower anorectal region and enlarged perirectal lymph nodes, but showed no evidence of distant metastases. We determined that radical resection would be difficult because of the retroperitoneal adherence of lymph nodes. Therefore, the patient received immunotherapy with pembrolizumab for nine cycles, which reduced the size of the perirectal lymph node (Fig. 2). The patient subsequently underwent robotic-assisted APR and lymph node dissection. The resected specimen showed a circumferentially elevated multilobulated tumor, measuring $11.5 \times 5.5 \mathrm{~cm}^{2}$, situated at the anorectal junction (Fig. 3). Histopathological examination showed spindle-shaped atypical melanocytes with acidophilic changes indicative of tumor cell necrosis in the rectal mucosa and tumor cell invasion of the superficial muscle layer (Fig. 4). Immunohistochemically, the tumor cells tested positive for SOX 10 and melan-A, thereby confirming the diagnosis of AMM (Fig. 4). We found no viable tumor cells in all 48 lymph nodes that were resected, and resection margins were tumor free. Despite immunotherapy-induced adrenal insufficiency, the patient was able to complete 15 cycles of adjuvant immunotherapy with pembrolizumab and remained relapse free at the 2-year postoperative follow-up.

Fig. 1. Colonoscopic image showed an elevated tumorous lesion within the lower rectum.

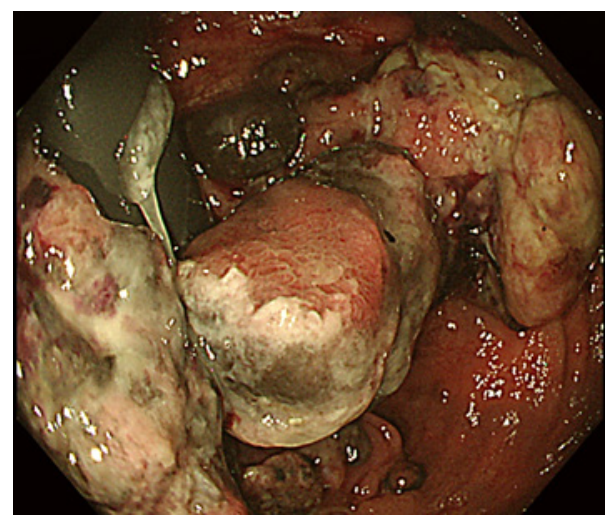



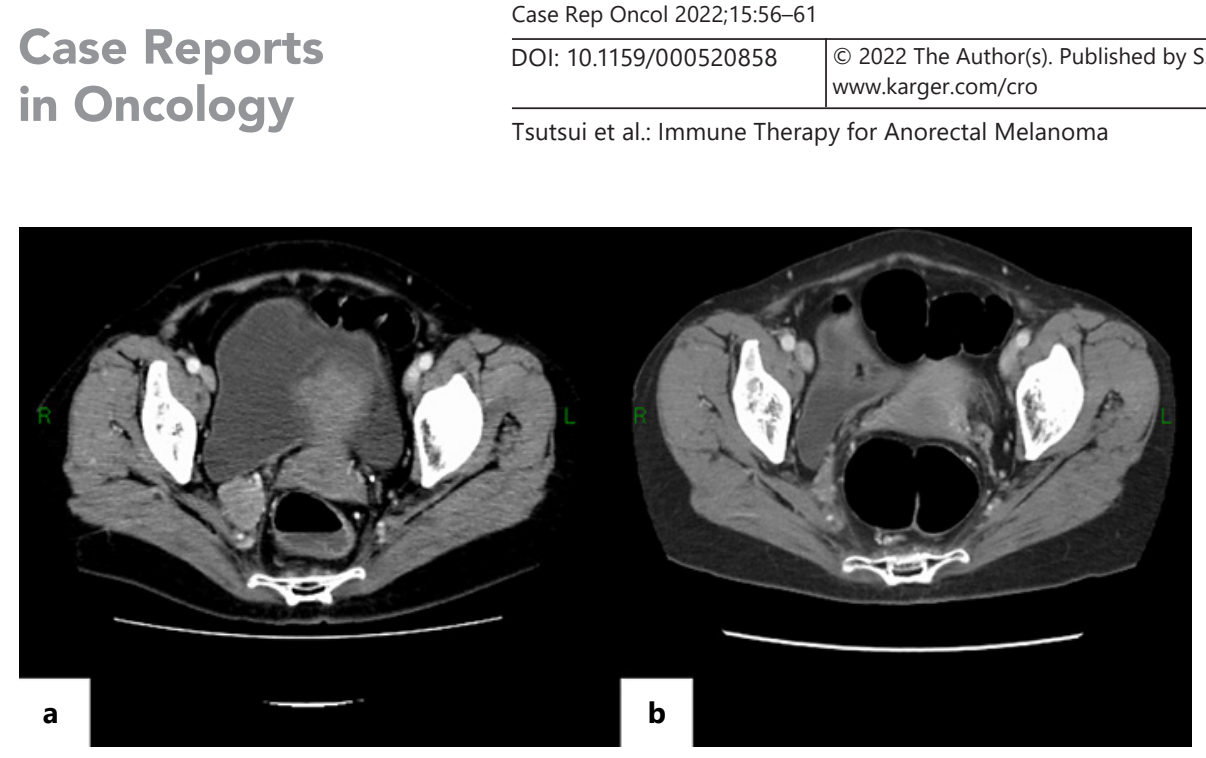

Fig. 2. CT scanning images of the patient that were obtained before (a) and after (b) immunotherapy. CT, computed tomography.

Fig. 3. The resected specimen showed a circumferentially elevated multilobulated tumor.

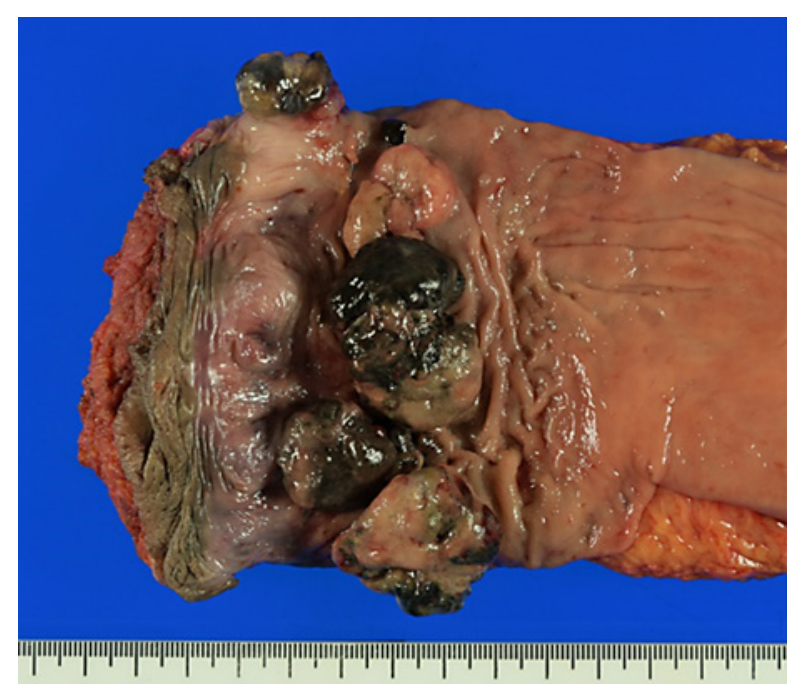

\section{Discussion}

AMM is a rare type of melanoma that accounts for $0.4-1.6 \%$ of total malignant melanomas $[4,5]$. AMM is commonly reported to women and is occurring more and more frequently in recent years [6]. AMM lesions are often mistaken for benign anal rectal lesions such as hemorrhoids and adenomatous polyps. Most patients are diagnosed with AMM when they have already developed distant metastasis or LNM. Weinstock reported that $41 \%$ of AMM cases had LNM and $22 \%$ had distant metastasis, while $37 \%$ had local disease [6]. The prognosis for AMM is considerably poorer worse than the prognosis for the more common cutaneous melanoma. AMM is a highly lethal disease, with a 5 -year survival rate of $6-22 \%[7,8]$. The TNM staging for head and neck mucosal melanoma has been validated, but no TNM staging is available for AMM. Nagarajan et al. [9] used a modified cutaneous melanoma staging system to predict the survival of patients with AMM. Patients with AMM and LNM were classified as having stage III cancer, with a 5-year survival rate of 18\% [9]. In general, complete surgical resection is the preferred therapeutic approach for resectable AMM, and APR or wide local excision (WLE) is performed. However, no comparative trials have been conducted to establish differences in survival between APR and WLE. Much of the published literature is limited to retrospective analyses. Previous 


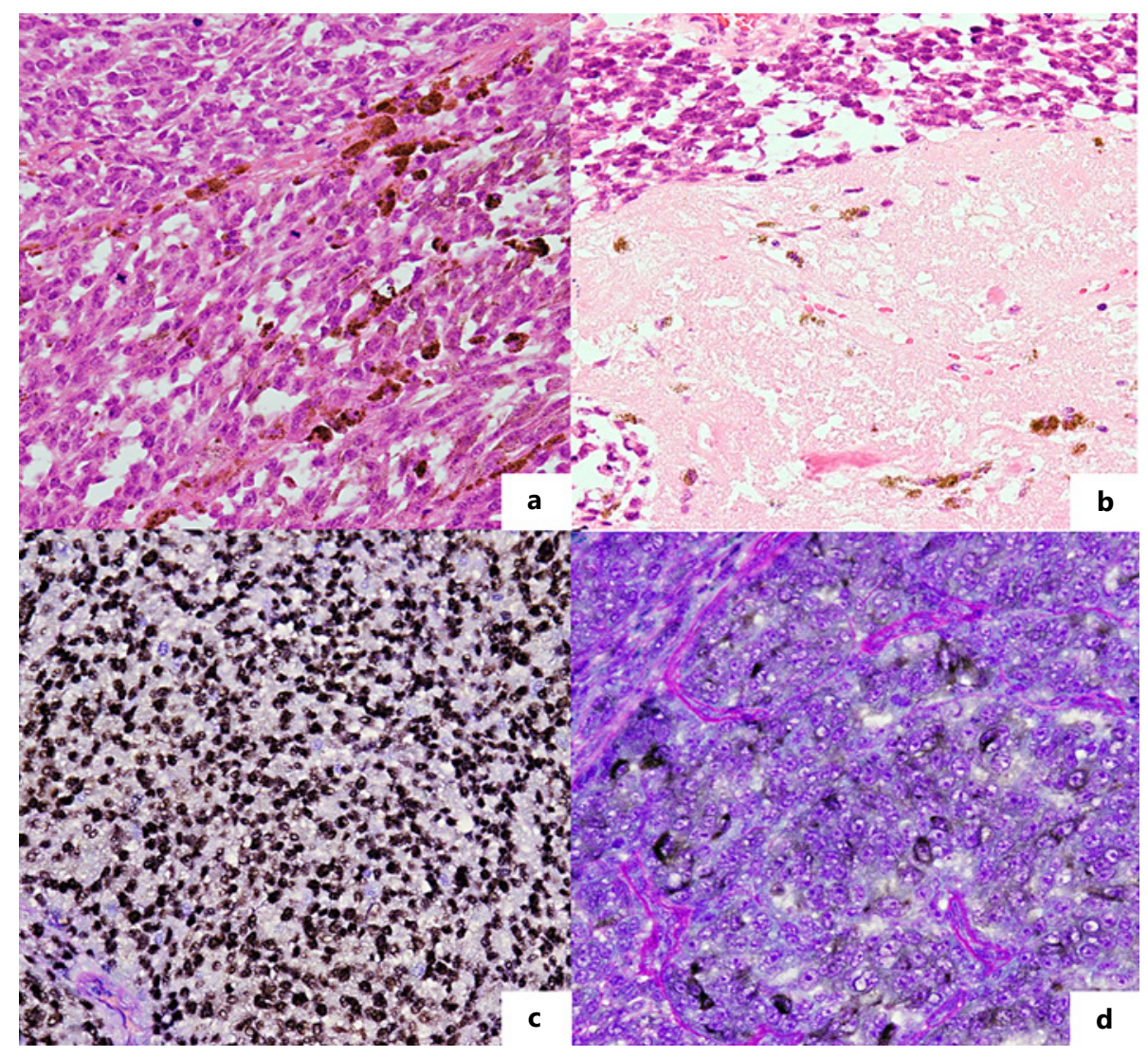

Fig. 4. Histopathological examination showed spindle-shaped atypical melanocytes (a) with acidophilic changes (b) indicative of tumor cell necrosis in the rectal mucosa (original magnification, $\times 200$ ). Immunohistochemistry showed that tumor cells expressed SOX 10 (c) and melan A (d) (original magnification, $\times 200$ ).

studies showed no significant difference in survival between patients treated with APR and WLE, and the indications for APR are controversial [10]. However, the abovementioned studies are not fully validated for immunotherapy, which could significantly influence the prognosis of AMM patients. However, it is important to consider that WLE has no benefits when the tumor is invading the sphincter complex or causing chronic bleeding or obstruction. APR should be performed on large tumors, such as that in our case, where WLE is technically impossible.

Conventionally, cytotoxic agents such as dacarbazine have been used for metastatic malignant melanoma. However, it is hard to say that these therapies have improved prognosis. Recently, immune-checkpoint inhibitors have been developed and a few of these have been approved for chemotherapy of malignant melanoma. Shoushtari et al. [11] described the efficacy of immunotherapy on mucosal melanoma, including AMM. In our patient, the pretreatment imaging findings which indicated a large LNM predicted a poor prognosis, although APR and lymph node dissection could ensure complete tumor resection. Moreover, we considered that radical resection would prove challenging because of the retroperitoneal adherence of lymph nodes. Due to the prediction of poor prognosis, an APR was planned for complete resection only if immunotherapy was effective. We administered pembrolizumab because there was no BRAF mutation in the resected specimen. As computed tomography imaging confirmed tumor size reduction and pathological response, it is expected that immunotherapy would be effective for preventing postoperative tumor recurrence. Adjuvant immunotherapy is recommended for head and neck mucosal melanoma and may be effective in treating AMM, which has a high risk 
of recurrence [12]. The patient remained recurrence free at 2-year follow-up after surgery, suggesting that postoperative adjuvant therapy with immunotherapy was effective.

\section{Conclusion}

In conclusion, preoperative immunotherapy and subsequent tumor resection allows early examination of on-treatment tumor tissue, which provides evidence of treatment response and important information for decisions on adjuvant treatment. Despite the very limited evidence, a combination of complete surgical resection and immunotherapy is expected to improve outcomes in AMM patients if immunotherapy is effective.

\section{Acknowledgments}

This work was partly supported by the National Cancer Center Research and Development Fund (29-A-3 and 2020-J-3). We would like to thank Editage (www.editage.com) for English language editing.

\section{Statement of Ethics}

This report complies with human research guidelines and contains evidence that the research was conducted ethically in accordance with the World Medical Association's Declaration of Helsinki. A written informed consent was obtained from the patient for this case report and the publication of accompanying images. This study is exempt from Institutional Review Board approval because single patient case reports are allowed to be reported without institutional review board approval.

\section{Conflict of Interest Statement}

The authors have no conflicts of interest to declare.

\section{Funding Sources}

This study did not receive funding.

\section{Author Contributions}

All authors contributed substantially to the conceptualization, drafting, and revisions of this manuscript.

\section{Data Availability Statement}

All data generated or analyzed during this study are included in this article. Further inquiries can be directed to the corresponding author.

\section{Karger ${ }^{\prime}=$}




\section{References}

1 Blecker D, Abraham S, Furth EE, Kochman ML. Melanoma in the gastrointestinal tract. Am J Gastroenterol. 1999;94(12):3427-33.

2 Malaguarnera G, Madeddu R, Catania VE, Bertino G, Morelli L, Perrota RE, et al. Anorectal mucosal melanoma. Oncotarget. 2018;9(9):8785-800.

3 Tokuhara K, Nakatani K, Tanimura H, Yoshioka K, Kiyohara T, Kon M. A first reported case of metastatic anorectal amelanotic melanoma with a marked response to anti-PD-1 antibody nivolumab: a case report. Int J Surg Case Rep. 2017;31:188-92.

4 Belli F, Gallino GF, Lo Vullo S, Mariani L, Poiasina E, Leo E. Melanoma of the anorectal region: the experience of the National Cancer Institute of Milano. Eur J Surg Oncol. 2009;35(7):757-62.

5 Pessaux P, Pocard M, Elias D, Duvillard P, Avril MF, Zimmerman P, et al. Surgical management of primary anorectal melanoma. Br J Surg. 2004;91(9):1183-7.

6 Weinstock MA. Epidemiology and prognosis of anorectal melanoma. Gastroenterology. 1993;104(1):174-8.

7 Meguerditchian AN, Meterissian SH, Dunn KB. Anorectal melanoma: diagnosis and treatment. Dis Colon Rectum. 2011;54(5):638-44.

8 Nam S, Kim CW, Baek SJ, Hur H, Min BS, Baik SH, et al. The clinical features and optimal treatment of anorectal malignant melanoma. Ann Surg Treat Res. 2014;87(3):113-7.

9 Nagarajan P, Piao J, Ning J, Noordenbos LE, Curry JL, Torres-Cabala CA, et al. Prognostic model for patient survival in primary anorectal mucosal melanoma: stage at presentation determines relevance of histopathologic features. Mod Pathol. 2020;33(3):496-513.

10 Kiran RP, Rottoli M, Pokala N, Fazio VW. Long-term outcomes after local excision and radical surgery for anal melanoma: data from a population database. Dis Colon Rectum. 2010;53(4):402-8.

11 Shoushtari AN, Munhoz RR, Kuk D, Ott PA, Johnson DB, Tsai KK, et al. Efficacy of anti-PD-1 agents in acral and mucosal melanoma. Cancer. 2016;122(21):3354-62.

12 Nenclares P, Ap Dafydd D, Bagwan I, Begg D, Kerawala C, King E, et al. Head and neck mucosal melanoma: the United Kingdom national guidelines. Eur J Cancer. 2020;138:11-8. 